\begin{tabular}{|c|l|}
\hline Title & Lipoxygenase inhibitors derived from marine macroalgae \\
\hline Author(s) & Kurihara, Hidey uki; Kagawa, Y oshio; Konno, Remi; Kim, Sang Moo; Takahashi, Koretaro \\
\hline Citation & $\begin{array}{l}\text { Bioorganic \& medicinal chemistry letters, 24(5), 1383-1385 } \\
\text { https://doi.org/10.1016/.bmcl.2014.01.046 }\end{array}$ \\
\hline Issue Date & 201403-01 \\
\hline Doc URL & http://hdl.handle.net/2115/55254 \\
\hline Type & article (author version) \\
\hline File Information & manuscriptLOXIRev1.pdf \\
\hline
\end{tabular}

Instructions for use 


\section{Lipoxygenase inhibitors derived from marine macroalgae}

${\text { Hideyuki Kurihara }{ }^{\mathrm{a},} \text {, Yoshio Kagawa }}^{\mathrm{a}}$, Remi Konno ${ }^{\mathrm{a}}$, Sang Moo Kim ${ }^{\mathrm{b}}$, Koretaro Takahashi ${ }^{\mathrm{a}}$

${ }^{a}$ Faculty and Graduate School of Fisheries Sciences, Hokkaido University, Minato, Hakodate, Hokkaido 041-8611, Japan

${ }^{b}$ Department of Marine Bioscience and Technology, Gangneung-Wonju National University, Gangneung 210-702, Korea

* Corresponding author. Tel.: +81 138405561 .

E-mail address: kuri@fish.hokudai.ac.jp 


\section{Abstract}

The solvent extracts from the algae Sargassum thunbergii (Sargassaceae) and Odonthalia corymbifera (Rhodomelaceae) were subjected to soybean lipoxygenase inhibitory screening. Two hydrophobic inhibitors were obtained from the extracts of $S$. thunbergii through inhibitory assay-guided fractionation. The inhibitors were identified as known exo-methylenic alkapolyenes $(6 Z, 9 Z, 12 Z, 15 Z)-1,6,9,12,15$-henicosapentaene and $(6 Z, 9 Z, 12 Z, 15 Z, 18 Z)-1,6,9,12,15,18$-henicosahexaene (2). The alkapolyenes $\mathbf{1}$ and $\mathbf{2}$ showed higher inhibitory activity than the known inhibitor nordihydroguaiaretic acid (NDGA). Pheophytin a (3) was obtained from the extract of $O$. corymbifera. The inhibitor $\mathbf{3}$ also showed higher inhibitory activity than NDGA. This is the first report on lipoxygenase inhibition of exo-methylenic alkapolyenes and a chlorophyll a-related substance.

Key words: lipoxygenase; inhibitor; Sargassum thunbergii; alkapolyene; Odonthalia corymbifera; pheophytin a. 
Lipoxygenase (LOX) catalyzes hydroperoxidation of unsaturated fatty acids. This reaction is a trigger to generate various leukotrienes, responsible for inducing psoriasis, asthma, rhintis, and arthritis, in arachidonate cascade. ${ }^{1}$ Inhibition of LOX is expected to prevent these diseases. LOX inhibitors, such as fatty acids, polyacetylenes, phenols, have been isolated from many terrestrial plants. ${ }^{2,3}$ Nordihydroguaiaretic acid (NDGA), a symmetric phenylpropanoid dimer, is a well-known LOX inhibitor obtained from Zygophyllaceae plant. ${ }^{4}$ This plant was used as a traditional medicine and nutritional supplement. However, renal and hepatotoxicity was disclosed during chronic use of this plant and NDGA. ${ }^{5}$ Thus it is expected to disclose a novel type of LOX inhibitor.

Many studies have been carried out on highly unsaturated fatty acids, leukotrienes, and LOX activity originated from macroalgae, ${ }^{6-9}$ whereas a few studies were performed on LOX inhibitors. ${ }^{10,11}$ During our screening of enzyme inhibitors derived from algal extracts, we disclosed potent LOX inhibitory activity of the extracts from the brown alga Sargassum thunbergii and the red alga Odonthalia corymbifera. In the present study, we isolated two exo-methylenic alkapolyenes from $S$. thunbergii and a chlorophyll a-related substance from $O$. corymbifera.

S. thunbergii (2.85 kg, air-dried) was collected at the coast of Otobe, south-western Hokkaido, Japan. Fresh alga washed with tap water was extracted with $\mathrm{MeOH}-$ water $(9: 1, \mathrm{v} / \mathrm{v})$ for a week. LOX inhibitory activity was assayed by a modified absorptiometry method ${ }^{12-14}$ for the extract and at a series of separation steps. An ethyl acetate-soluble fraction $(8.46 \mathrm{~g})$ partitioned from the extract (69.5 g) was chromatographed on silica gel column with hexane-EtOAc (3:1, v/v) as eluent. Further chromatography was employed on silica gel column with $n$-hexane-EtOAc (19:1, v/v). The active fraction was twice separated on preparative thin-layer chromatography with $n$-hexane-EtOAc (3:1, v/v) and $100 \%$ hexane as developing solvent to afford inhibitors 1 (7.9 $\mathrm{mg}, 2.8 \mathrm{ppm}$ yield) and $\mathbf{2}$ (2.8 $\mathrm{mg}, 1.1 \mathrm{ppm}$ yield). Inhibitors 1 and 2 were identified as $(6 Z, 9 Z, 12 Z, 15 Z)-1,6,9,12,15$-henicosapentaene ${ }^{15}$ (HEP, Figure 1) and $(6 Z, 9 Z, 12 Z, 15 Z, 18 Z)-$ $-1,6,9,12,15,18$-henicosahexaene ${ }^{14}(\mathrm{HEH}$, Figure 1$)$, respectively, by their spectral data, ${ }^{16}$ compared with the literature data. ${ }^{17-20}$ Stereostructures of double bonds of $\mathbf{1}$ and $\mathbf{2}$ were deduced to be all $Z$ configurations from the ${ }^{13} \mathrm{C}$ NMR data $(\delta$ 25.63-25.64 for 1 ; $\delta 25.52-25.64$ for 2 ) for the allylic positions in 1,4-pentadiene moieties of them. Pfeffer et al. ${ }^{21}$ reported that the ${ }^{13} \mathrm{C}$ NMR data of $\delta 25.7,30.5$, and 35.7 for the allylic positions in 1,4-pentadiene moieties of polyunsaturated fatty acid residues were assigned as Z,Z-, Z,E-, and E,E-isomers, respectively. HEP (1) and HEH (2) inhibited soybean LOX in concentration-dependent manners (Figure 2). The IC $_{50}$ values of 1 and 2 were calculated 40 $\mu \mathrm{M}$ and $5.0 \mu \mathrm{M}$, respectively. Both of $\mathbf{1}$ and $\mathbf{2}$ exhibited higher LOX inhibitory activity than NDGA $\left(\mathrm{IC}_{50}=400 \mu \mathrm{M}\right)$.

Exo-methylenic alkapolyenes $\mathbf{1}$ and 2, first isolated from the brown alga Fucus vesiculosus in $1971,^{15}$ are widely distributed in brown algae, except for the ones from the red alga Bryocladia cuspidata. ${ }^{22}$ HEH (2) was previously isolated from $S$. thunbergii. ${ }^{23}$ To our knowledge, there was no report on enzyme inhibitory activity of $\mathbf{1}$ and $\mathbf{2}$. Their 
oxidative degradation products were identified as characteristic odoriferous compounds of brown algae. ${ }^{24}$ In the present study, they were stable in the air for several weeks (data not shown). Soybean lipoxygenase mediates oxygenation at the 6th (and 9th) carbon atoms from the methyl end group of linoleic, docosahexaenoic, eicosapentaenoic, $\alpha$-linolenic, and arachidonoc acids, while it mediates no oxygenation in the case of 2-methylarachidonic acid. ${ }^{25}$ Thus the lipoxygenase may recognize fatty acids as substrate with at the carboxyl end. The alkapolyenes $\mathbf{1}$ and $\mathbf{2}$ are different structures from carboxyl groups of fatty acids. This difference is speculated to be responsible for inhibition of the lipoxygenase.

O. corymbifera (790 g, air-dried) was collected at the coast of Hakodate, south-western Hokkaido, Japan. Fresh seaweed washed with tap water was extracted with acetone-water (95:5, v/v) for 3 days. An ethyl acetate-soluble fraction (7.72 g) partitioned from the extract (51.4 g) was chromatographed on silica gel column with chloroform-MeOH $(9: 1, \mathrm{v} / \mathrm{v})$ as eluent. Silica gel column chromatography was employed again with $n$-hexane-acetone (3:1, v/v). The active fraction was separated on preparative thin-layer chromatography with chloroform-MeOH (99:1, v/v) as developing solvent to afford an inhibitor 3 (6.5 $\mathrm{mg}, 8.2 \mathrm{ppm}$ yield). The inhibitor $\mathbf{3}$ was identified as pheophytin a ${ }^{26}$ (Figure 1) by comparison with the authentic pheophytin a (Wako Pure Chemical Industries, Ltd., Osaka, Japan) on silica gel TLC and spectroscopic data ${ }^{27}$ with literature ones. ${ }^{28,29}$ Inhibitory activity of $\mathbf{3}$ was compared with its related compounds, pyropheophytin a (4, Tama Biochemical Co., Ltd., Tokyo, Japan), pheophorbide a (5, Wako Pure Chemical Industries, Ltd, Osaka, Japan), and pyropheophorbide a (6, Tama Biochemical Co., Ltd., Tokyo, Japan) (Table 1). Compounds 3 and 4 which possessed a phytyl group showed higher inhibitory activity than the other chlorophyll a-related compounds and NDGA. Hydrophobic phytyl group could enhance the inhibitory effect against LOX.

Pheophytin a, the primary electron acceptor in photosystem II, is a demetalation product of chlorophyll a through biological or non-biological processes. ${ }^{30}$ Chlorophyll a is commonly distributed in algae. Hence, various algae are capable of containing pheophytin a, which was detected in all extracts of two green algae, three brown algae, and four red algae by qualitative TLC analysis (data not shown). However, only the extract of $O$. corymbifera showedinhibition against LOX among the extracts examined. The reason why inhibitory acrivity of the extracts derived from the other algae was not detected is supposed that inhibitory activity of pheophytin a is easily hindered or covered with coexistent other consituents. In the case of $O$. corymbifera extract, other constituent could be occasionally hindered the inhibitory activity. Pheopytin a has been reported to have various activities. ${ }^{26,31-33}$ Nevertheless, this study is the first report on LOX inhibition of pheophytin a.

In conclusion, we isolated soybean LOX inhibitors as alkapolyenes $\mathbf{1}$ and $\mathbf{2}$ from the brown alga S. thunbergii and pheophytin a (3) from the red alga O. corymbifera. They inhibited the enzyme more strongly than the well-known inhibitor NDGA. 


\section{Acknowledgements}

We thank to Dr. Eri Fukushi and Mr. Yusuke Takata, GC-MS \& NMR Laboratory, Faculty of Agriculture, Hokkaido University, for measurements of MS and NMR spectra. We are also grateful to JSPS-KOSEF Cooperative Research Program, Regional Innovation Strategy Support Program of the MEXT Japan, and the Hokusui Society Foundation for their financial supports.

\section{References and notes}

1

2

3

4

5

6

Bell, R. L.; Young, P. R.; Albert, D.; Lanni, C.; Summers; J. B., Brooks, D. W.; Rubin, P.; Carter, G. W. Int. J. Immunopharmacol., 1992, 14, 505.

Schneider, I.; Bucar, F., Phytother. Res., 2005, 19, 81.

Schneider, I.; Bucar, F., Phytother. Res., 2005, 19, 263.

Whitman, S.; Gezginci,M.; Timmermann, B. N.; Holman, T. R. J. Med. Chem., 2002, 45, 2659.

Artega, S.; Andrade-Cetto, A.; Cardenas, R. J. Ethnopharmacol., 2005, 98, 231.

Andreou, A.; Brodhun, F.; Feussner, I. Prog. Lipid Res., 2009, 48, 148.

Bernart, M. W.; Gerwick, W. H. Phytochemistry, 1994, 36, 1233.

Sajiki, J.; Kakimi, H. J Chromatogr., A, 1998, 795, 227.

Sajiki, J. Jpn. J. Toxicol. Environ. Health, 1999, 45, 100.

Matsukawa, R.; Dubinsky, A.; Kishimoto, E.; Masaki, K.; Masuda, Y.; Takeuchi, T.; Chihara, M.; Yamamoto, Y.; Niki, E. ; Karube, I.; J. Appl. Phycol., 1997, 9, 29.

Shibata, T.; Nagayama, K.; Tanaka, R.; Yamaguchi, K.; Nakamura, T. J. Appl. Phycol., 2003, 61. Anthon, G. E.; Barrett, D. M. J. Agric. Food Chem., 2001, 49, 32.

Komoda, T.; Yoshida, K.; Abe, N.; Sugiyama, Y.; Imachi, M.; Hirota, H.; Koshino, H.; Hirota; A. Biosci., Biotechnol., Biochem., 2004. 68: 104.

Solutions A and B, and substrate solution were previously prepared as follows. Solution A was $20 \mathrm{mM}$ 3-dimethylaminobenzoic acid in $100 \mathrm{mM}$ borate buffer (pH 9.0). Solution B was 10 mM 3-methyl-2-benzothiazolinone hydrazone and $5 \mathrm{mg} / \mathrm{mL}$ hemoglobin (Wako Pure Chemical Industries, Ltd., 
Osaka, Japan) in $50 \mathrm{mM}$ phosphate buffer ( $\mathrm{pH}$ 5.0). Substrate solution was prepared bu adding linoleic acid (Wako Pure Chemical Industries, Ltd., Osaka, Japan, $140 \mathrm{mg}$ ) and Tween 20 (Wako Pure Chemical Industries, Ltd., Osaka, Japan, $280 \mathrm{mg})$ in $1 \mathrm{~N} \mathrm{NaOH}(0.6 \mathrm{~mL})$ and water $(5 \mathrm{~mL})$ and mixing by using a Pasteur pipette. The mixture was made up to $20 \mathrm{~mL}$ by adding water. This substrate solution was divided into 2 mL-aliquots, flushed with argon gas, and stored at $-30^{\circ} \mathrm{C}$ until use.

A test sample dissolved in methanol $(0.02 \mathrm{~mL})$ was added into solution $\mathrm{A}(0.91 \mathrm{~mL})$ and $0.4 \mathrm{mg} / \mathrm{mL}$ soybean LOX (Sigma) $100 \mathrm{mM}$ borate buffer ( $\mathrm{pH} 9.0)$ solution $(0.02 \mathrm{~mL})$. This solution was pre-incubated at $27.5^{\circ} \mathrm{C}$ for $5 \mathrm{~min}$. LOX reaction was initiated by adding the substrate solution $(0.05 \mathrm{~mL})$ incubated at $27.5^{\circ} \mathrm{C}$ for $5 \mathrm{~min}$, and terminated by adding solution $\mathrm{B}(1 \mathrm{~mL})$ to form colored product. After $5 \mathrm{~min}$, the reaction mixture was added $1 \%$ sodium dodecyl sulfate $(1 \mathrm{~mL})$ and mixed vigorously. The absorbance at $598 \mathrm{~nm}$ was compared with that in the control test.

Halsall, T. G.; Hills, I. R. J. Chem. Soc. D, Chem. Commun., 1971, 448.

(6Z,9Z,12Z,15Z)-1,6,9,12,15-Henicosapentaene (HPE, 1), EI-MS m/z: $286\left(\mathrm{M}^{+}\right)$, EI-HR-MS m/z: 286.2622 (calculated 286.2661 for $\left.\mathrm{C}_{21} \mathrm{H}_{34}\right),{ }^{1} \mathrm{H}-\mathrm{NMR}\left(500 \mathrm{MHz}, C D C l_{3}\right)$ ppm: 5.80 (m, 1H, H-2), 5.35 (m, 8H, H-6, 7, 9, $10,12,13,15,16), 5.00(\mathrm{dd}, J=1.4,17.1 \mathrm{~Hz}, 1 \mathrm{H}, \mathrm{H}-1), 4.90(\mathrm{dd}, J=1.4,10.0 \mathrm{~Hz}, 1 \mathrm{H}, \mathrm{H}-1), 2.80(\mathrm{~m}, 6 \mathrm{H}, \mathrm{H}-8$, 11, 14), 2.05 (m, 6H, H-3, 5, 17), 1.45 (m, 2H, H-4), 1.28 (m, 6H, H-18, 19, 20), 0.87 (t, J = 6.8 Hz, 3H, H-21), ${ }^{13} \mathrm{C}-\mathrm{NMR}\left(125 \mathrm{MHz}, \mathrm{CDCl}_{3}\right.$ ) ppm: 138.74 (C-2), 130.47, 129.93, 128.52, 128.39, 128.03, 127.99, 127.93, $127.55(\mathrm{C}-6,7,9,10,12,13,15,16), 114.50$ (C-1), 33.31 (C-3), 31.51 (C-19), 29.32 (C-18), 28.84 (C-4), 27.20 (C-17), 26.65 (C-5), 25.64, 25.64, 25.63 (C-8, 11, 14), 22.56 (C-20), 14.04 (C-21). (6Z,9Z,12Z,15Z,18Z)-1,6,9,12,15,18-Henicosahexaene (HHE, 2), EI-MS m/z: $284\left(\mathrm{M}^{+}\right)$, EI-HR-MS m/z: 284.2480 (calculated 284.2504 for $\left.\mathrm{C}_{21} \mathrm{H}_{32}\right),{ }^{1} \mathrm{H}-\mathrm{NMR}\left(500 \mathrm{MHz}, C D C l_{3}\right)$ ppm: 5.80 (m, 1H, H-2), 5.35 (m, 8H, H-6, 7, 9, 10, 12, 13, 15, 16, 18, 19), 5.00 (dd, $J=1.0,17.0 \mathrm{~Hz}, 1 \mathrm{H}, \mathrm{H}-1), 4.94(\mathrm{dd}, J=1.0,10.1 \mathrm{~Hz}, 1 \mathrm{H}, \mathrm{H}-1), 2.81$ (m, 8H, H-8, 11, 14, 17), 2.06 (m, 6H, H-3, 5, 20), 1.44 (m, 2H, H-4), $0.96(\mathrm{t}, J=7.5 \mathrm{~Hz}, 3 \mathrm{H}, \mathrm{H}-21),{ }^{13} \mathrm{C}-\mathrm{NMR}(125 \mathrm{MHz}$ $C D \mathrm{Cl}_{3}$ ) ppm: $138.74(\mathrm{C}-2), 132.03,129.96,128.55,128.45,128.19,128.16,127.95,127.95,127.89,127.01$ (C-6, 7, 9, 10, 12, 13, 15, 16, 18, 19), 114.50 (C-1), 33.30 (C-3), 28.83 (C-4), 26.65 (C-5), 25.64, 25.61, 25.61, $25.52(\mathrm{C}-8,11,14,17), 20.54(\mathrm{C}-20), 14.25(\mathrm{C}-21)$. Youngblood, W. W.; Blumer, M. Mar. Biol., 1973, 21, 163.

18 Holmeide, A, K.; Skattebol, L.; Sydnes, M. J. Chem. Soc., Perkin Trans. 1, 2001, 1942.

19 Wright, J. L. C. Phytochemistry, 1980, 19, 143.

20 Gregson, R. P.; Kazlauskasm R.; Murphy, P. T.; Wells, R. J. Aust. J. Chem., 1977, 30, 2527. 
Pfeffer, P. E.; Luddy, F. E.; Unruh, J. J. Am. Oil Chem. Soc., 1977, 54, 380.

Caccamese, S.; Rinehart, Jr., K. L. Experientia, 1978, 34, 1129.

Qin, M.; Li, M.; Yin, S.; Wang, C.; Wang, B. Haiyang Kexue, 2007, 31, 47.

Broekhof, N. L. J. M.; Witteveen, J. G.; Van der Weerdt, A. J. A. Recl. Trav. Chim. Pays-Bas., 1986, $105,436$. Holman, R. T. Egwim, P. O, Christie, W. W. J. Biol. Chem., 1969, 244, 1149.

Okai, Y.; Higashi-Okai, K. J. Sci. Food Agric., 1997, 74, 531.

Pheophytin a (3), VIS $\lambda_{\max }$ nm: 666, 608, 535, 506, 410; FD-MS m/z: $870.5\left(\mathrm{M}^{+}\right)$, FD-HR-MS m/z: 870.5639 (calculated 870.5659 for $\left.\mathrm{C}_{55} \mathrm{H}_{74} \mathrm{~N}_{4} \mathrm{O}_{5}\right),{ }^{1} \mathrm{H}-\mathrm{NMR}\left(500 \mathrm{MHz}, C D C l_{3}\right)$ ppm: 9.499 (s, 1H, H-10), 9.364 (s, $1 \mathrm{H}$, H-5) 8.534 (s, 1H, H-20), 7.975 (dd, $J=12.0,17.8 \mathrm{~Hz}, 1 \mathrm{H}, \mathrm{H}-3 \mathrm{a}), 6.270$ (d, $J=17.8 \mathrm{~Hz}, 1 \mathrm{H}, \mathrm{H}-3 \mathrm{~b}), 6.243$ (s, 1H, H-13b), 6.151 (d, $J=12.0 \mathrm{~Hz}, 1 \mathrm{H}, \mathrm{H}-3 \mathrm{~b}), 5.109$ (m, 1H, phytyl), 4.468 (m, 1H, H-18), 4.444 (m, $1 \mathrm{H}$, H-17), 4.276 (m, 1H, phytyl), 4.191 (m, 1H, phytyl), 3.860 (s, 3H, H-13d), 3.666 (s, 3H, H-12a), 3.634 (m, $2 \mathrm{H}$, H-8a), 3.381 (s, 3H, H-7a), 3.214 (s, 3H, H-2a), 2.830, 2.731, 2.457, 2.341 (m, 4H, Hs-17a and 17b), 1.850 (m, 2H, phytyl), 1.783 (d, $J=7.41 \mathrm{~Hz}, 3 \mathrm{H}, \mathrm{H}-18 \mathrm{a}), 1.677$ (t, $J=7.58 \mathrm{~Hz}, 3 \mathrm{H}, \mathrm{H}-8 \mathrm{~b}), 1.600$ (m, 2H, phytyl), 1.552 (s, 3H, phytyl), 1.480 (m, 1H, phytyl), 1.290 (m, 2H, phytyl), 1.234 (br. s, 14H, phytyl), 0.827 (d, $J=6.56 \mathrm{~Hz}$ $6 \mathrm{H}$, phytyl), 0.781 (d, $J=6.65 \mathrm{~Hz}, 3 \mathrm{H}$, phytyl), 0.761 (d, $J=6.55 \mathrm{~Hz}, 3 \mathrm{H}$, phytyl), ${ }^{13} \mathrm{C}-\mathrm{NMR}(125 \mathrm{MHz}$, $C D C_{3}$ ) ppm: 189.64 (C-13a), 172.93 (C-17c), 172.22 (C-13c), 169.90 (C-19), 161.25 (C-16), $155.66(\mathrm{C}-6)$, 150.94 (C-9), 149.64 (C-14), 145.16 (C-8), 142.82 (phytyl), 142.03 (C-1), 137.94 (C-11), 136.28 (C-3), 136.17 (C-4), 135.84 (C-7), 131.85 (C-2), 129.10 (C-3a), 129.05 (C-12), 128.98 (C-13), 122.78 (C-3b), 117.71 (phytyl), 105.14 (C-15), 104.43 (C-10), 97.54 (C-5), 93.11 (C-20), 64.69 (C-13b), 61.45 (phytyl), 53.32 (C-17), 50.95 (C-13d), 50.33 (C-18), 39.78, 39.34, 37.37, 37.31, 37.24, 36.2, 32.74, 32.60 (phytyl), 29.69 (C-17a), 29.69 (C-17b), 27.94, 24.97, 24.62, 24.40 (phytyl), 23.06 (C-18a), 22.69, 22.60, 19.70, 19.64 (phytyl), 19.46 (C-8a), 17.41 (C-8b), 16.27 (phytyl), 12.09 (C-2a), 12.09 (C-12a), 11.24 (C-7a).

Milenkovic, S. M.; Zvezdanovic, J. B.; Andelkovic, T. D.; Markovic, D. Z. Adv. Technol. 2012, 1, 16.

Chaves, O. S.; Gomes, R. A.; Tomaz, A. C. A.; Fernandes, M. G.; Junior, L. G. M.; Agra, M. F.; Braga, V. A.; Souza, M. F. V. Molecules, 2013, 18, 2769.

Saga, Y.; Tamiaki, H. Chem. Biodiversity, 2012, 9, 1659.

Lanfer-Marquez, U. M.; Barros, R. M. C.; Sinnecker, B. P. Food Res. Int., 2005, 38, 885.

Ina, A.; Hayashi, K.; Nozaki, H.; Kamei, Y. Int. Dev. Neurosci., 2007, 25, 63.

Subramoniam, A.; Asha, V. V.; Nair, S. A.; Sasidharan, S. P. Sureshkumar, P. K.; Rajendran, K. N.; Karunagaran, D.; Ramalingam, K. Inflammation, 2012, 35, 959. 


\section{Figure captions}

Fig. 1. Exo-methylenic alkapolyenes $\mathbf{1}$ and $\mathbf{2}$ and pheophytin a (3) as lipoxygenase inhibitors. Compounds 4-6 are pheophytin a-related compounds.

Fig. 2. Inhibition of $\mathbf{1}$ and $\mathbf{2}$ against soybean lipoxygenase. Open circle: 1, open box: 2, closed triangle: nordihydroguaiaretic acid (positive control). 


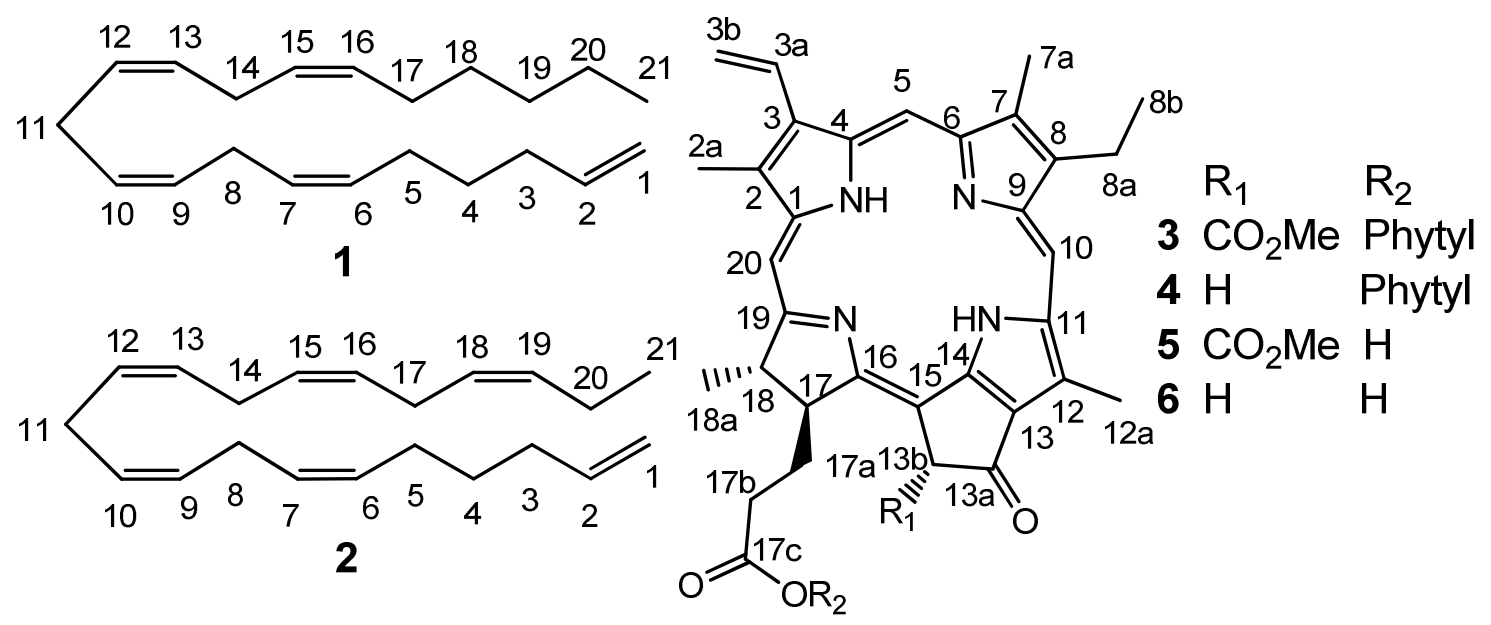

Fig. 1 


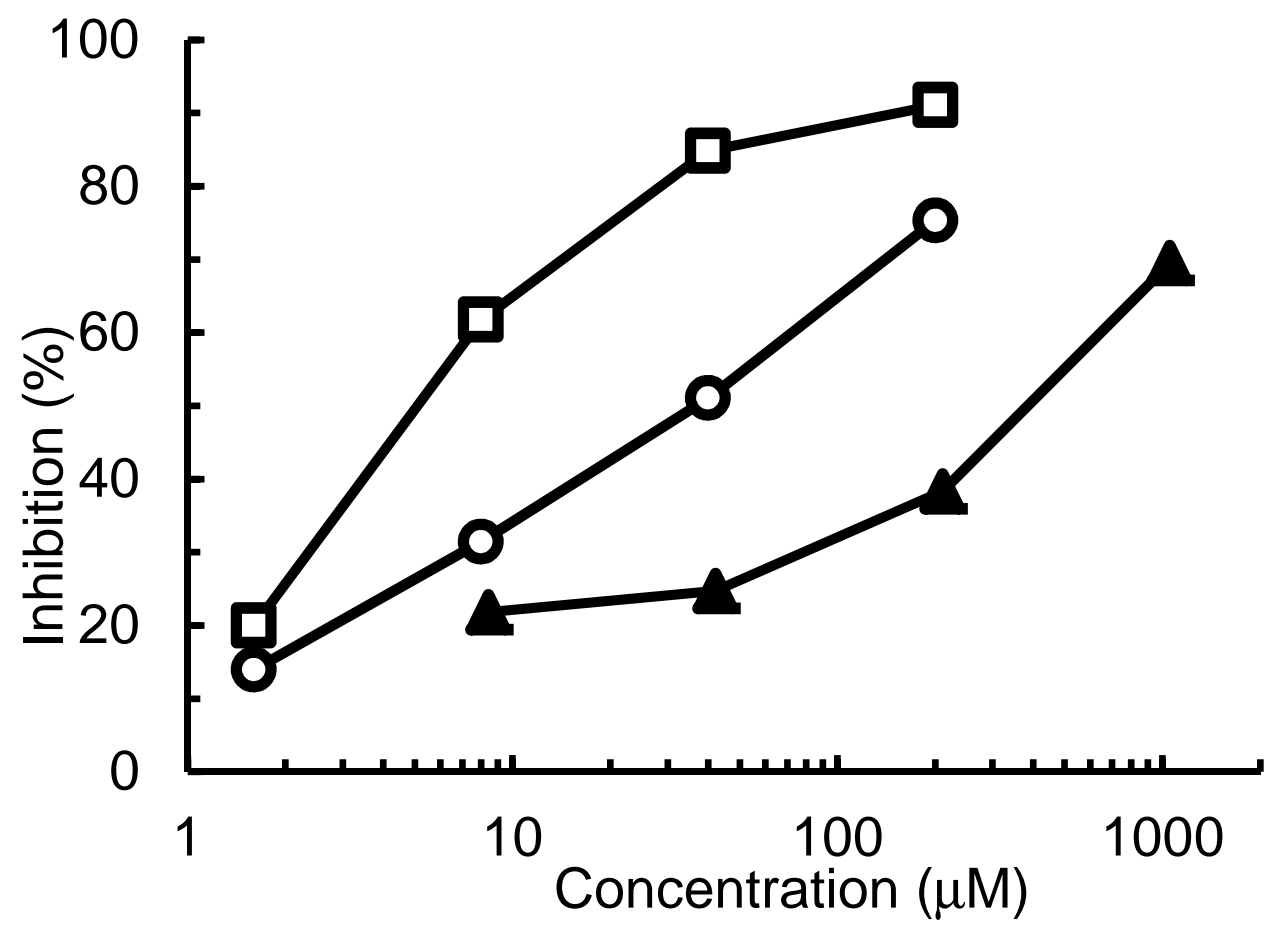

Fig. 2 


\section{Table 1}

Inhibition of pheophytin a (3) and related compounds

(4-6) against soybean lipoxygenase ${ }^{a}$

Compound Inhibition $(\%)($ mean $\pm \mathrm{SE}, \mathrm{n}=3)$

3

$37.6 \pm 2.3^{b}$

4

$29.4 \pm 2.5^{b}$

5

$6.5 \pm 1.3^{\mathrm{b}}$

6

$15.0 \pm 1.2$

NDGA

$12.7 \pm 0.5$

${ }^{\mathrm{a}}$ Test concentration was $100 \mu \mathrm{M}$.

${ }^{\mathrm{b}}$ Significant difference vs NDGA by Student's $t$ test $(p<0.05)$. 
Graphic abstract

Lipoxygenase inhibitors derived from marine macroalgae

Hideyuki Kurihara", Yoshio Kagawa, Remi Konno, Sang Moo Kim, Koretaro Takahashi

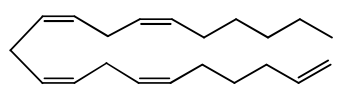

1

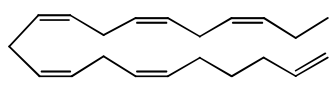

2

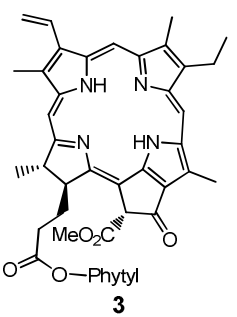

